\title{
EXAMINATION FOR INFLUENZA IgA AND IgM ANTIBODIES IN PREGNANCIES ASSOCIATED WITH FETAL NEURAL-TUBE DEFECTS
}

\author{
Mary J. WarRell, J. O'H. TObin and N. J. WalD* \\ Virology Laboratory, John Radcliffe Hospital, Oxford OX3 9DU and the *ICRF Cancer \\ Epidemiology and Clinical Trials Unit, Radcliffe Infirmary, Oxford OX2 $6 \mathrm{HE}$
}

SUMmary. Early antenatal serum specimens were collected from 25 women who subsequently gave birth to infants with anencephaly or spina bifida. Fluorescentantibody tests for influenza-specific IgA and IgM antibodies, although shown to be reliable for the detection of recent influenzal infection, failed to demonstrate such infection in any of these women. Early infection was detected in one of 50 control women who gave birth to a healthy infant. The results indicate that influenzal infection in pregnancy was not a major cause of neural-tube defects in Oxford during 1972-76, if a cause at all.

\section{INTRODUCTION}

The role of influenza in the causation of fetal neural-tube defects is unclear. Early investigations were based upon only a clinical history of an influenza-like illness in the mother during pregnancy. Thus, Coffey and Jessop (1959), in a study after the 1957-58 epidemic in Ireland, recognised neural-tube defects 3.5 times more commonly among infants born to women with such a history than among infants born to women without. Saxén, Klemetti and Härö (1974), in a case-control study of congenital malformations in Finland during 1964-71, obtained a history of influenzal illness during the first trimester three times more often from women who gave birth to infants with neural-tube defects than from women who produced healthy infants; this difference was much reduced in the case of illnesses occurring in the second trimester and disappeared with third-trimester illnesses. Pleydell (1960) enquired into the occurrence of influenza-like illness among pregnant women during an epidemic in Northamptonshire; one of 43 "ill" mothers produced an infant with a neural-tube defect $(2 \cdot 3 \%)$, and only two of 1040 mothers without symptoms of influenza did so $(0 \cdot 2 \%)$. However, this apparently large difference was not statistically significant. Campbell (1953) and Doll, Bradford-Hill and Sakula (1960), too, were unable to confirm any association between influenza-like illness in women during pregnancy and neural-tube defects in their infants. In an epidemiological study, which did not rely on the reporting of an influenza-like illness, Hakosalo and Saxén (1971) demonstrated a prevalence of neural-tube defects 2.6 times higher among infants born to women who conceived during the 1957 epidemic than among infants born to women who conceived shortly before or after the epidemic. On the other hand, Leck (1963), in a similar study in Birmingham, found no increase in the prevalence of neural-tube defects after the epidemics of 1957, 1959 and 1961. Unfortunately, in none of the above studies could the possibility be excluded that some other factor, related to the influenzal illness, such as pyrexia or an antipyretic drug, was the actual teratogen.

Studies in which the maternal illness was confirmed as influenza serologically, by haemagglutination-inhibition or complement-fixation tests, have not provided any more convincing evidence of an aetiological association with neural-tube defects. None of these was able to demonstrate that the influenzal infection occurred early enough in pregnancy to affect develop-

Received 27 May 1980; accepted 1 Jul 1980.

Requests for reprints should be sent to Dr N. J. Wald. 
ment of the neural tube (the human neural tube normally closes at about 6 weeks), either because the serum specimens were collected too late (Walker and McKee, 1959), or because the antibody levels were generally too low (Wilson et al., 1959; Hardy et al., 1961).

The study reported here was an attempt to detect the occurrence of influenza infection in the early months of pregnancy in women who had given birth to infants with anencephaly or spina bifida, by examination of previously collected antenatal sera for the presence of influenza-specific IgA and IgM antibodies. These are relatively short-lived antibodies, persisting for only 3-12 months and 8-16 weeks, respectively, after infection (De Silva, 1972; Urquhart, 1974; Buchner et al., 1977). Initial tests were made of the sensitivity of the fluorescent-antibody technique for detecting these antibodies, in non-pregnant patients with confirmed influenza.

\section{MATERIALS AND METHODS}

Patients. a). Forty-two serum specimens were collected from 14 non-pregnant medical students and nurses from whom influenza $A$ virus had been isolated, during 1973 and 1974. The sera were taken at intervals from 2 days before to 40 weeks after isolation of virus from the patient's throat, and were stored at $-40^{\circ} \mathrm{C}$. They were matched, as far as possible, with sera collected, usually within the same month as each test serum, from 14 control subjects in whom recent influenzal infection was excluded by standard serological methods. To eliminate bias, the test and control sera were coded and mixed before examination for IgM and IgA antibodies. b). A serum sample collected within the first 4 months of pregnancy was available from 12 women who had given birth to anencephalic infants, and from 13 who had given birth to infants with spina bifida. Each sample was compared with two control sera collected, with one exception, within a month of the test serum, from women of matched gestational age and parity, and who had produced healthy infants. The test and control sera were taken during 1972-76, and were stored at $-40^{\circ} \mathrm{C}$ until tested in 1979; they were coded and mixed before testing.

Fluorescent-antibody (FA) technique. This was essentially the method described by De Silva et al. (1973). Trypsinised Vero cells were suspended, at a concentration of $6 \times 10^{6} \mathrm{cells} / \mathrm{ml}$ in Medium 199 (Wellcome Reagents) containing $2 \%$ fetal-calf serum. Sufficient tissue-culturegrown influenza virus, either strain A/Eng/929/73 (supplied by Dr M. S. Pereira, Public Health Laboratory Service, Colindale) or strain B/Eng/2287/76, was added to infect about $10 \%$ of the cells. After mixing in a roller for 20 min, drops of cell suspension were distributed on to 6-mm-diameter spaces on teflon-coated microscope slides. These were then incubated in a candlebox for $20-24 \mathrm{~h}$ at $35^{\circ} \mathrm{C}$. The resulting monolayers were fixed in acetone and the slides were stored at $-20^{\circ} \mathrm{C}$. The FA test was performed by placing test or control sera, at dilutions of 1 in 3 and 1 in 9 , on the slide spots and incubating at $37^{\circ} \mathrm{C}$, for $45 \mathrm{~min}$ to detect IgA and $105 \mathrm{~min}$ to detect IgM. After washing, anti-human IgA (Hyland Laboratories) or anti-human IgM (Wellcome Reagents) fluorescein conjugate was added for $30 \mathrm{~min}$ at $37^{\circ} \mathrm{C}$. After further washing, the monolayers were examined in a fluorescence microscope with epi-illumination. Each test was controlled with known positive and negative sera on the same slide. Sera giving positive results for IgM were tested by the latex slide test for rheumatoid factor to exclude the possibility of non-specific fluorescence. The presence of influenza-specific IgM was confirmed by repeating the FA test on the IgM fraction of the serum, separated either by ultracentrifugation on sucrose density gradients or by adsorption with staphylococcal protein $A$. The absence of contaminating IgG and IgA in the sucrose-gradient fractions was checked by immunodiffusion. Specific immunofluorescence was confined to the IgM-containing fractions.

\section{RESULTS}

\section{Detection of specific IgM and IgA in non-pregnant patients with influenza}

The table illustrates the frequency of detection of specific $\operatorname{IgM}$ and $\operatorname{IgA}$ in the 14 patients at various intervals after infection with influenza $A$ virus. From 1 to 6 weeks after virus isolation, IgA was present in $100 \%$ of the specimens examined and IgM in $62 \%$. After 6 weeks, the 
TABLE

Presence of specific IgM and IgA antibodies in 14 patients with influenza A, at intervals after infection

\begin{tabular}{|c|c|c|}
\hline \multirow{2}{*}{$\begin{array}{c}\text { Interval after } \\
\text { infection* (weeks) }\end{array}$} & \multicolumn{2}{|c|}{$\begin{array}{l}\text { Number of sera } \\
\text { containing/number } \\
\text { examined for } \\
\text { virus-specific }\end{array}$} \\
\hline & IgM & IgA \\
\hline $\begin{array}{c}<1 \\
1-6 \\
6-11 \\
11-16 \\
16-21 \\
21-31 \\
31-40\end{array}$ & $\begin{array}{l}0 / 1 \dagger \\
5 / 8(62 \%) \\
1 / 14(7 \%) \\
2 / 11(18 \%) \\
0 / 4 \quad \\
1 / 2(50 \%) \\
0 / 2\end{array}$ & $\begin{array}{ll}0 / 1 \dagger & \\
8 / 8 & (100 \%) \\
9 / 14 & (64 \%) \\
7 / 11 & (64 \%) \\
3 / 4 & (75 \%) \\
1 / 2 & (50 \%) \\
2 / 2 & (100 \%)\end{array}$ \\
\hline
\end{tabular}

* Calculated from day of isolation of virus from patient's throat. † Serum specimen collected 2 days before virus isolation.

frequency of detection of IgM declined markedly, but IgA persisted, in most cases, for at least 40 weeks. Tests for specific IgA and IgM antibodies were negative in all 24 sera collected from the 14 control subjects. Influenza B-specific antibody was not detected in any specimen.

\section{Pregnant patients}

The periods of gestation at the time of blood collection, in the 25 women who produced abnormal infants, ranged from 68 to 111 days, with a median of 92 days; there was no clustering of conceptional dates, which were spread evenly throughout each of the 4 years of the study. In none of the 25 mothers was IgA or IgM, specific for either influenza A or B, detected. Among the 50 control mothers, with normal infants, one showed the presence of influenza A-specific IgA and IgM; this patient was bled in February 1975.

\section{Discussion}

Blood was obtained from 25 mothers carrying fetuses with neural-tube defects within 4 months of the onset of conception. Although specific $\operatorname{IgA}$ was shown to persist in the majority of patients with influenza for as long as $\mathbf{4 0}$ weeks and to be detectable by the FA technique even after prolonged storage of serum specimens at $-40^{\circ} \mathrm{C}$, evidence of influenzal infection was not found in any of the 25 women. The only recent infection recognised in the pregnancy study was among the 50 control women carrying healthy babies. The abnormal pregnancies were evenly spread over the four years from 1972 until 1976. Although these were not major epidemic years for influenza in the United Kingdom, the lowest attack rate, during these years, among the 15-64-year olds was 2\% (Public Health Laboratory Service Standing Advisory Committee on Influenza, 1977, and Dr N. D. Noah, personal communication).

Our results, therefore, indicate that neither influenza A nor B infection was a major cause of neural-tube defects in Oxford from 1972 to 1976. Because of the low infection rate in the community and the small number of patients available for study, influenza cannot be entirely ruled out as a cause of neural-tube defects, but it would seem to be unlikely to account for more than $5 \%$ of cases.

\section{REFERENCES}

Buchner, Y. I., Heath, R. B., Collins, J. V. and Pattison, J. R. 1977. Serum IgM antibody and influenza A infection. J. clin. Path., 30, 723. 
CAMPbell, W. A. B. 1953. Influenza in early pregnancy-effects on the foetus. Lancet, 1, 173.

COFFEY, V. P. AND Jessop, W. J. E. 1959. Maternal influenza and congenital deformities, a prospective study. Lancet, 2, 935.

DE Silva, G. P. L. M. 1972. Studies on specific IgG, IgA and IgM fluorescent antibody levels as an aid to the rapid diagnosis of certain viral infections. Ph.D thesis, Manchester University.

De Silva, L. M., Khan, M. S., Kampfner, G., Tobin, J. O’H., Gillett, R. and Morris, C. A. 1973. The post-mortem diagnosis of influenzal infection by fluorescent IgG, IgA and IgM antibody studies on necropsy blood. J. Hyg., Camb., 71, 107.

Doll, R., Bradford-Hill, A. AND Sakula, J. 1960. Asian influenza in pregnancy and congenital defects. Br. J. prev. soc. Med., 14, 167.

Hakosalo, J. AND SAXÉn, L. 1971. Influenza epidemic and congenital defects. Lancet, 2, 1346.

Hardy, J. M. B., Azarowicz, E. N., Mannini, A., Medearis, D. N. and CoOKe, R. E. 1961. The effect of Asian influenza on the outcome of pregnancy, Baltimore 1957-58. Am. J. publ. Hlth, 51, 1182.

LECK, I. 1963. The incidence of malformations following influenza epidemics. Br. J. prev. soc. Med., 17, 70.

Pleydell, M. J. 1960. Anencephaly and other congenital abnormalities. An epidemiological study in Northamptonshire. Br. med. J., $1,309$.

Public Health laboratory Service Standing Advisory Committee on Influenza. 1977. Influenza surveillance 1972-1975. J. Hyg., Camb., 78, 223.

SAXÉn, L., KLEMETTI, A. AND HÄRÖ, A. S. 1974. A matched-pair register for studies of selected congenital defects. Am. J. Epidem., 100, 297.

Urquhart, G. E. D. 1974. Serum IgM and IgA responses in influenza A infections. J. clin. Path., 27, 198.

WALKER, W. M. AND MCKeE, A. P. 1959. Asian influenza in pregnancy-relationship to fetal anomalies. Obstet. Gynec., N.Y., 13, 394.

Wilson, M. G., Heins, H. L., Imagawa, D. T. and Adams, J. M. 1959. Teratogenic effects of Asian influenza. J. Am. med. Ass., 171, 638. 